PROCEEDINGS OF THE

AMERICAN MATHEMATICAL SOCIETY

Volume 131, Number 6, Pages 1891-1899

S 0002-9939(02)06733-3

Article electronically published on November 4, 2002

\title{
SOME REMARKS ON LIOUVILLE TYPE RESULTS FOR QUASILINEAR ELLIPTIC EQUATIONS
}

\author{
E. N. DANCER AND YIHONG DU
}

(Communicated by David S. Tartakoff)

\begin{abstract}
For a wide class of nonlinearities $f(u)$ satisfying $f(0)=f(a)=0, f(u)>0$ in $(0, a)$ and $f(u)<0$ in $(a, \infty)$,

we show that any nonnegative solution of the quasilinear equation $-\Delta_{p} u=$ $f(u)$ over the entire $\mathbb{R}^{N}$ must be a constant. Our results improve or complement some recently obtained Liouville type theorems. In particular, we completely answer a question left open by Du and Guo.
\end{abstract}

\section{INTRODUCTION}

Consider the semilinear diffusion equation

$$
u_{t}=\Delta u+f(u), x \in \mathbb{R}^{N}, t \in[0, \infty),
$$

where $f$ is a $C^{1}$ function satisfying

$$
f(0)=f(a)=0, f(u)>0 \text { in }(0, a) \text { and } f(u)<0 \text { in }(a, \infty) .
$$

This problem arises in population biology and chemical reaction theory, and has attracted extensive study; we refer to $\mathrm{AW}$ and the references therein for more details of the background.

If

$$
\underline{\lim }_{u \rightarrow 0^{+}} \frac{f(u)}{u^{1+2 / N}}>0,
$$

then the so-called hair-trigger effect of Aronson and Weinberger AW implies that for any solution $u(x, t) \in[0, a]$ with $u(x, 0) \not \equiv 0$, one has $\lim _{t \rightarrow \infty} u(x, t)=a$ uniformly for $x \in \mathbb{R}^{N}$. Moreover, it is shown in [AW] that the exponent $1+2 / N$ is sharp in the sense that the hair-trigger effect fails if $1+2 / N$ is replaced by any $\xi>1+2 / N$ in the above condition.

A simple consequence of the above hair-trigger effect is an interesting Liouville type result for the corresponding elliptic problem, namely, for such $f(u)$, the equation

$$
-\Delta u=f(u), x \in \mathbb{R}^{N},
$$

Received by the editors February 8, 2002.

2000 Mathematics Subject Classification. Primary 35J15, 35J60.

Key words and phrases. Quasilinear elliptic equation, Liouville theorem, sweeping principle.

The work of the first author was partially supported by the Australian Research Council.

(C)2002 American Mathematical Society 
has only the constant solutions $u=0$ and $u=a$ satisfying $0 \leq u \leq a$. Note that since $f(u)<0$ in $(a, \infty)$, it can be shown that any globally bounded nonnegative solution of (1.1) satisfies $0 \leq u \leq a$; moreover, with certain further conditions for $f(u)$ near infinity, for example, $\underline{\lim }_{u \rightarrow+\infty} \frac{-f(u)}{u^{q}} \in(0, \infty]$ for some $q>1$, it can be shown that any nonnegative solution of (1.1) must satisfy $0 \leq u \leq a$ (see [DM]).

One would like to check the sharpness of the exponent $1+2 / N$ for this Liouville type result. For $N>2$ and any $\xi>N /(N-2)$, one can find a $C^{1}$ function $f(u)$ such that $f(0)=f(a)=0, f(u)>0$ on $(0, a), f(u)<0$ for $u>a$, and $f(u)=c u^{\xi}$ for small positive $u$, such that $-\Delta u=f(u)$ has a positive solution satisfying $0<u<a$ and it decays to zero at infinity. Indeed, if $\xi \in(N /(N-2),(N+2) /(N-2))$, then such an example was constructed near the end of the paper Da. If $\xi \geq$ $(N+2) /(N-2)$, it is well known that $-\Delta u=u^{\xi}$ has a bounded positive solution $u_{\xi}$ which decays to 0 at infinity, and the required example can be easily constructed by making use of this fact.

So the problem reduces to checking whether (1.1) has a nonconstant solution satisfying $0 \leq u \leq a$ when $f(u)$ behaves like $c u^{\xi}$ near 0 with $\xi \in(1+2 / N, N /(N-2)]$, the gap left open above.

The present note addresses this question and related problems. In particular, we will show that the answer to the above question is negative.

Theorem 1.1. Suppose that $f(u)$ is a $C^{1}$ function satisfying

$$
f(0)=f(a)=0, f(u)>0 \forall u \in(0, a), \varliminf_{u \rightarrow 0^{+}} f(u) / u^{N /(N-2)} \in(0,+\infty] .
$$

Then any solution of (1.1) satisfying $0 \leq u \leq a$ must be a constant.

This result and the examples mentioned above imply that the critical exponent for the Liouville theorem is $N /(N-2)$ instead of $1+2 / N$.

Theorem 1.1 will follow as a consequence of one of our general results on solutions of $p$-Laplacian equations of the type

$$
-\Delta_{p} u=c(x) f(u) \text { in } \mathbb{R}^{N}(N \geq 2)
$$

where $\Delta_{p} u=\operatorname{div}\left(|D u|^{p-2} D u\right), p>1, c \in L^{\infty}\left(\mathbb{R}^{N}\right)$ and $0<c_{1}<c(x)<c_{2}<\infty$. By a solution of (1.2) we mean a function $u \in C^{1}\left(\mathbb{R}^{N}\right)$ satisfying

$$
\int_{\mathbb{R}^{N}}|D u|^{p-2} D u \cdot D \psi d x=\int_{\mathbb{R}^{N}} c(x) f(u) \psi d x, \forall \psi \in C_{0}^{\infty}\left(\mathbb{R}^{N}\right) .
$$

The detailed statements of our main results (Theorem 2.1 and Theorem 2.4) and their proofs are contained in the next section. These results improve or complement recent Liouville type theorems obtained in [BP, DG, $\overline{\mathrm{DM}}, \mathrm{SZ}]$. In particular, Theorem 2.1 completely answers a question left open in [DG]. Our proofs are based upon recent results of Bidaut-Veron and Pohozaev [BP] and Serrin and Zou [SZ].

\section{MAIN RESUlts AND PROOFS}

We say that $f(s)$ is locally quasi-monotone on $[0, \infty)$ if for any bounded interval $\left[s_{1}, s_{2}\right] \subset[0, \infty)$, there exists a continuous increasing function $L(s)$ such that $f(s)+$ $L(s)$ is nondecreasing in $s$ for $s \in\left[s_{1}, s_{2}\right]$. Clearly, this condition is less restrictive than requiring $f(s)$ to be locally Lipschitz continuous on $[0, \infty)$. 
Our first main result is the following.

Theorem 2.1. Let $f(s)$ be continuous and locally quasi-monotone on $[0, \infty)$ and satisfy the following conditions:

$\left(F_{1}\right)$ For some $a>0$,

$$
f(0)=f(a)=0, f(s)>0 \text { in }(0, a), f(s)<0 \text { in }(a, \infty) .
$$

$\left(F_{2}\right)$ If $N \geq p$, we assume further that for some small $\delta>0$ and $\xi \in\left(0, \frac{(p-1) N}{N-p}\right]$ when $N>p, \xi \in(0, \infty)$ when $N=p$, there exists a constant $\sigma>0$ such that

$$
f(s) \geq \sigma s^{\xi}, \forall s \in(0, \delta) .
$$

Then any solution of (1.2) satisfying $0 \leq u \leq a$ is a constant.

Remark 2.2. (i) It is shown in DG that if $f(s)$ satisfies $\left(F_{1}\right)$, then any globally bounded nonnegative solution of (1.2) satisfies $0 \leq u \leq a$; moreover, if for some $q>p-1$,

$$
\varliminf_{s \rightarrow \infty} \frac{-f(s)}{s^{q}} \in(0, \infty],
$$

then any nonnegative solution of (1.2) satisfies $0 \leq u \leq a$.

(ii) The example in Remark 1.2 (iii) of [DG] shows that when $N>p$, for any $\xi>(p-1) N /(N-p)$, one can find $c(x)$ satisfying $0<c_{1} \leq c(x) \leq c_{2}<\infty$ such that $-\Delta_{p} u=c(x) u^{\xi}$ has a positive solution which decays to 0 at infinity. This implies that our condition $\left(F_{2}\right)$ in Theorem 2.1 is sharp.

(iii) Theorem 2.1 positively answers the conjecture in DG, Remark 1.2 (iv)]. Note that Theorem 1.1 follows from Theorem 2.1 by letting $p=2$.

The main ingredient for the proof of Theorem 2.1 is the following recent result due to Bidaut-Veron and Pohozaev [BP] and Serrin and Zou [SZ, Theorem II].

Proposition 2.3. $\quad$ (i) If $N<p, \Omega \subset \mathbb{R}^{N}$ is an exterior domain in $\mathbb{R}^{N}$ (i.e., $\left\{x \in \mathbb{R}^{N}:|x|>R\right\} \subset \Omega$ for some $\left.R>0\right)$, and $u \in C^{1}(\Omega)$ is nonnegative and satisfies (in the weak sense) $-\Delta_{p} u \geq 0$, then $u$ must be a constant.

(ii) If $N \geq p, \Omega \subset \mathbb{R}^{N}$ is an exterior domain, and $u \in C^{1}(\Omega)$ is nonnegative and satisfies (in the weak sense) $-\Delta_{p} u \geq u^{\xi}$ with $\xi \in(0,(p-1) N /(N-p)]$ when $N>p, \xi \in(0, \infty)$ when $N=p$, then $u \equiv 0$.

Proof of Theorem 2.1. By part (i) of Proposition 2.3, we need only consider the case that $N \geq p$. So we assume $N \geq p$ from now on.

Since $-\Delta_{p} u \geq 0$ and $u \geq 0$, by the strong maximum principle (see $\mathrm{Va}$ ] or $\mathrm{PS}$, Theorem 1]), either $u \equiv 0$ or $u>0$ everywhere. Therefore we assume $u>0$. We need to show that $u \equiv a$.

Let us observe that it suffices to show $u(x) \rightarrow a$ as $|x| \rightarrow \infty$. Indeed, if this is true but $u \not \equiv a$, then $\inf _{x \in \mathbb{R}^{N}} u(x)<a$ is achieved at some $x_{0} \in \mathbb{R}^{N}$. Therefore the function $v(x)=u(x)-u\left(x_{0}\right)$ satisfies

$$
v \geq 0, v\left(x_{0}\right)=0,-\Delta_{p} v=-\Delta_{p} u \geq 0 .
$$

By the strong maximum principle we deduce $v \equiv 0$, that is, $u(x) \equiv u\left(x_{0}\right)$. But this is a contradiction to the assumption that $u(x) \rightarrow a$ as $|x| \rightarrow \infty$. 
To show that $u(x) \rightarrow a$ as $|x| \rightarrow \infty$, we choose $R>0, \alpha \in\left(0, \min _{|x|=R} u(x)\right)$ and consider the boundary value problem

$$
\begin{array}{clrl}
-\Delta_{p} w & =c_{1} f(w) & & \text { when } R<|x|<R_{n}, \\
w & =\alpha & & \text { when }|x|=R, \\
w & =0 & & \text { when }|x|=R_{n},
\end{array}
$$

where $\left\{R_{n}\right\}$ is an increasing sequence of numbers satisfying $R<R_{1}, R_{n} \rightarrow \infty$ as $n \rightarrow \infty$. For each $n \geq 1, w=u$ is a super solution to (2.1) while $w=0$ is a sub-solution. Hence by the well known sub- and super-solution method (see [D]), (2.1) has a minimal and maximal solution satisfying $0 \leq w \leq u$. Let $w_{n}$ denote the minimal solution. We observe that $w_{n}$ must be radially symmetric as it is minimal and problem (2.1) is invariant under rotations around the origin. Furthermore, $w_{n+1}$ is a super-solution to (2.1) while 0 is a sub-solution. Hence (2.1) has a solution between 0 and $w_{n+1}$. It follows that its minimal solution $w_{n}$ must satisfy $w_{n} \leq w_{n+1}$. Therefore, we have

$$
w_{n}(x) \leq w_{n+1}(x) \leq u(x) \text { when } R<|x|<R_{n}, n=1,2, \ldots .
$$

It follows that $w(x):=\lim _{n \rightarrow \infty} w_{n}(x)$ is well-defined for $|x|>R$ and $0 \leq w(x) \leq$ $u(x)$.

Since $0 \leq c f\left(w_{n}\right) \leq c_{2} \max _{s \in[0, a]} f(s)$ and $0 \leq w_{n} \leq u \leq a$, for any given bounded smooth sub-domain $\Omega$ of $\left\{x \in \mathbb{R}^{N}:|x|>R\right\}$, applying standard regularity theory (see [To]) to the equation $-\Delta_{p} w_{n}=c f\left(w_{n}\right)$ for all large $n$ so that $\Omega \subset A_{n}:=$ $\left\{x \in \mathbb{R}^{N}: R<|x|<R_{n}\right\}$, we find that $\left\{\left.u_{n}\right|_{\Omega}\right\}$ is bounded in $C^{1, \alpha}(\Omega)$ for some $\alpha$. As $C^{1, \alpha}(\Omega)$ imbeds compactly into $C^{1}(\bar{\Omega}),\left\{\left.w_{n}\right|_{\Omega}\right\}$ has a convergent subsequence in $C^{1}(\bar{\Omega})$. It follows that $w_{n} \rightarrow w$ in $C^{1}(\bar{\Omega})$ for any bounded smooth domain $\Omega$ of $\left\{x \in \mathbb{R}^{N}:|x|>R\right\}$. Therefore, $w$ satisfies

$$
-\Delta_{p} w=c_{1} f(w) \text { when }|x|>R, w=\alpha \text { when }|x|=R .
$$

Since each $w_{n}$ is radially symmetric, so is $w: w(x)=w(r), r=|x|$. Hence we can write

$$
-\left(r^{N-1}\left|w^{\prime}\right|^{p-2} w^{\prime}\right)^{\prime}=r^{N-1} c_{1} f(w) \text { for } r>R, w(R)=\alpha .
$$

Denote $\phi(r)=r^{N-1}\left|w^{\prime}(r)\right|^{p-2} w^{\prime}(r)$. We find from the above equation that $\phi^{\prime}(r)=$ $-r^{N-1} c_{1} f(w(r)) \leq 0$ for $r>R$. Hence $\phi(r)$ is a nonincreasing function and there are two possibilities:

(i) $\phi(r)$ is negative for large $r$, or (ii) $\phi(r) \geq 0$ on $[R, \infty)$.

Case (i) cannot occur because otherwise, there exists $R_{0} \geq R$ such that $w^{\prime}(r)<0$ for $r>R_{0}$. Thus $w(r)<w\left(R_{0}+1\right)<w\left(R_{0}\right) \leq a$ for all $r>R_{0}+1$. Let $\alpha_{1}=w\left(R_{0}+1\right)$. Then we can use $\left(F_{1}\right)$ and $\left(F_{2}\right)$ to find some $\sigma_{1} \in(0, \sigma]$ such that $f(s) \geq \sigma_{1} s^{\xi}$ for $s \in\left(0, \alpha_{1}\right)$. Thus,

$$
-\Delta_{p} w=c_{1} f(w) \geq c_{1} \sigma_{1} w^{\xi}
$$

in the exterior domain $\Omega_{R_{0}+1}:=\left\{x \in \mathbb{R}^{N}:|x|>R_{0}+1\right\}$. We can now easily check that for $\beta=\left(c_{1} \sigma_{1}\right)^{-p}, v(x):=w(\beta x)$ is a positive solution of $-\Delta_{p} v \geq v^{\xi}$ in the exterior domain $\Omega_{\left(R_{0}+1\right) / \beta}$, contradicting Proposition 2.3.

So case (ii) must occur which implies that $w^{\prime}(r) \geq 0$ for $r>R$. Since $w \leq u \leq a$, $\alpha_{2}:=\lim _{r \rightarrow \infty} w(r)$ exists. If $\alpha_{2}<a$, then $w(r) \leq \alpha_{2}$ for all $r>R$ and we can use the same argument as in case (i) to deduce a contradiction to Proposition 2.3. Thus we necessarily have $\lim _{r \rightarrow \infty} w(r)=a$ and hence, due to $a \geq u(x) \geq w(x)$, $\lim _{|x| \rightarrow \infty} u(x)=a$, as we wanted. This finishes the proof. 
Let us note that while condition $\left(F_{1}\right)$ can be regarded as a kind of global restriction on $f(u)$, condition $\left(F_{2}\right)$ is local, it only restricts the behaviour of $f(u)$ near $u=0$. When condition $\left(F_{1}\right)$ is strengthened, it is possible to relax condition $\left(F_{2}\right)$ and still obtain some Liouville type theorems. Our second main result addresses this point.

Theorem 2.4. Suppose $N>p>1$ and $f(u)$ is a $C^{1}$ function satisfying $\left(F_{1}\right)$ and furthermore,

$\left(F_{3}\right)$ for some $\xi \in\left(0, \frac{N p}{N-p}-1\right)$ and all $u \in(0, a), \frac{d}{d u}\left[f(u) / u^{\xi}\right] \leq 0$,

$\left(F_{4}\right)$ for some small $\delta_{1}>0$,

$$
\int_{a-\delta_{1}}^{a}\left[\int_{s}^{a} f(t) d t\right]^{-1 / p} d s=\infty
$$

Then the only solutions to

$$
-\Delta_{p} u=f(u) \text { in } \mathbb{R}^{N}
$$

satisfying $0 \leq u \leq a$ are $u \equiv 0$ and $u \equiv a$.

Let us note that $f(u)=u^{\xi}-u^{q}$ satisfies $\left(F_{3}\right)$ whenever $\xi \in\left(0, \frac{N p}{N-p}-1\right)$ and $q>\xi$, but it satisfies $\left(F_{2}\right)$ only if $\xi \in\left(0, \frac{(N-1) p}{N-p}-1\right]$ and $q>\xi$.

Condition $\left(F_{4}\right)$ is satisfied when $f(a)=0$ and $0 \leq f(s) \leq M|s-a|^{p-1}$ for some $M>0$ and all $s \in\left(a-\delta_{1}, a\right)$. Thus, if $f$ is $C^{1}$, then $\left(F_{4}\right)$ is automatically satisfied provided that $1<p \leq 2$, but it is an extra restriction when $p>2$. We suspect that $\left(F_{4}\right)$ is unnecessary in Theorem 2.4 .

The main ingredients in our proof of Theorem 2.4 are the following two results.

Proposition 2.5. Suppose $N>p>1$. If the $C^{1}$ function $f(u)$ satisfies

(i) $f(0)=0, f(u) \geq 0$ for $u>0$,

(ii) $\frac{d}{d u}\left[f(u) / u^{\xi}\right] \leq 0$ for some $\xi \in\left(0, \frac{N p}{N-p}-1\right)$ and all $u>0$, and

(iii) $f(u) \geq u^{q}$ for some $q>p-1$ and all large $u$, then problem (2.2) has only the trivial solution $u \equiv 0$ in $\mathbb{R}^{N}$.

Proposition 2.6. Suppose that $N>p>1$. Let $f(s)$ be continuous and locally quasi-monotone on $[0, \infty)$. Suppose $f(s)$ satisfies $\left(F_{1}\right),\left(F_{4}\right)$ and

$\left(F_{2}^{\prime}\right)$ for some small $\delta>0$, there exist constants $\sigma>0$ and $\xi \in\left(p-1, \frac{N p}{N-p}-1\right)$ such that

$$
f(s) \geq \sigma s^{\xi}, \forall s \in(0, \delta) .
$$

Then for any solution of (1.2) satisfying $0 \leq u \leq a$, we have $\sup _{x \in \mathbb{R}^{N}} u(x)<a$ unless $u \equiv a$.

Proposition 2.5 is due to Serrin and Zou (see [SZ, Theorem II(c)]). To prove Proposition 2.6, we will make use of the following variant of a weak sweeping principle used in $[\mathrm{DG}$, which develops the idea of the well-known sweeping principle due to Serrin [S] for Laplacian equations.

Lemma 2.7 (Weak sweeping principle). For each $t \in[0,1]$, let $\zeta_{t}: \mathbb{R}^{N} \rightarrow \mathbb{R}^{N}$ be a diffeomorphism of $\mathbb{R}^{N}$ onto itself, satisfying $\zeta_{0}(x)=x$ and that $\zeta_{t}(x) \rightarrow \zeta_{t_{0}}(x)$ as $t \rightarrow t_{0}$ uniformly for $x$ in compact subsets of $\mathbb{R}^{N}$. Let $\mathcal{D}$ be a bounded smooth domain in $\mathbb{R}^{N}$ and $\mathcal{D}_{t}:=\zeta_{t}(\mathcal{D})$. Suppose that $h(x, s)$ is measurable in $x \in \mathbb{R}^{N}$, continuous and locally quasi-monotone (uniformly for $x$ in compact subsets of $\mathbb{R}^{N}$ ) with respect 
to $s \in(-\infty, \infty)$. Let $u_{t}$ and $v_{t}, t \in[0,1]$, be functions in $W^{1, p}\left(\mathcal{D}_{t}\right) \cap C\left(\overline{\mathcal{D}}_{t}\right)$ and satisfy in the weak sense, for some $\epsilon>0$,

$$
\begin{gathered}
-\Delta_{p} u_{t} \geq h\left(x, u_{t}\right)+\epsilon,-\Delta_{p} v_{t} \leq h\left(x, v_{t}\right) \text { in } \mathcal{D}_{t}, \forall t \in[0,1], \\
u_{t} \geq v_{t}+\epsilon \text { on } \partial \mathcal{D}_{t}, \forall t \in[0,1] .
\end{gathered}
$$

Moreover, suppose that $u_{t_{0}} \geq v_{t_{0}}$ in $\mathcal{D}_{t_{0}}$ for some $t_{0} \in[0,1]$, and $t \rightarrow u_{t} \circ \zeta_{t}$ and $t \rightarrow v_{t} \circ \zeta_{t}$ are continuous from $[0,1]$ to $C(\overline{\mathcal{D}})$. Then

$$
u_{t} \geq v_{t} \text { on } \mathcal{D}_{t}, \forall t \in[0,1]
$$

Proof. Denote

$$
T=\left\{t \in[0,1]: u_{t} \geq v_{t} \text { on } \mathcal{D}_{t}\right\}=\left\{t \in[0,1]: u_{t} \circ \zeta_{t} \geq v_{t} \circ \zeta_{t} \text { on } \mathcal{D}\right\} .
$$

Clearly $T$ is a closed set with $t_{0} \in T$. We show that $T$ is relatively open in [0,1], which implies $T=[0,1]$, as required.

Since $u_{t} \circ \zeta_{t}$ and $v_{t} \circ \zeta_{t}$ vary continuously with $t$, it is easily seen that there exist finite numbers $s_{1}<s_{2}$ such that $u_{t}(x), v_{t}(x) \in\left[s_{1}, s_{2}\right]$ for all $x \in \bigcup_{0 \leq t \leq 1} \mathcal{D}_{t}$ and all $t \in[0,1]$.

Since $h(x, s)$ is locally quasi-monotone in $s$, we can find a continuous increasing function $L(s)$ such that $\tilde{h}(x, s):=h(x, s)+L(s)$ is nondecreasing in $s$ for all $x \in$ $\bigcup_{0 \leq t \leq 1} \mathcal{D}_{t}$ and $s \in\left[s_{1}, s_{2}\right]$.

Let $\delta>0$ be sufficiently small. Then, for any $t \in T$,

$$
\begin{aligned}
-\Delta_{p} u_{t}+L\left(u_{t}\right) & \geq \tilde{h}\left(x, u_{t}\right)+\epsilon \geq \tilde{h}\left(x, v_{t}\right)+\epsilon \geq-\Delta_{p} v_{t}+L\left(v_{t}\right)+\epsilon \\
& \geq-\Delta_{p}\left(v_{t}+\delta\right)+L\left(v_{t}+\delta\right) \text { in } \mathcal{D}_{t},
\end{aligned}
$$

and

$$
u_{t} \geq v_{t}+\delta \text { on } \partial \mathcal{D}_{t} .
$$

By the weak maximum principle (see, e.g., D, Theorem 4.9]) we obtain $u_{t} \geq v_{t}+\delta$ in $\mathcal{D}_{t}$, i.e., $u_{t} \circ \zeta_{t} \geq v_{t} \circ \zeta_{t}+\delta$ in $\mathcal{D}$. Thus for all $\tau \in[0,1]$ with $|\tau-t|$ small, $u_{\tau} \circ \zeta_{\tau} \geq v_{\tau} \circ \zeta_{\tau}$ in $\mathcal{D}$. This shows that $T$ is relatively open in [0,1]. The proof is complete.

Remark 2.8. From the above proof, we immediately see that Lemma 2.7 remains true if $(2.3)$ is replaced by

$$
-\Delta_{p} u_{t} \geq h\left(x, u_{t}\right),-\Delta_{p} v_{t} \leq h\left(x, v_{t}\right)-\epsilon \text { in } \mathcal{D}_{t}, \forall t \in[0,1] .
$$

Proof of Proposition 2.6. Suppose $a=\sup _{x \in \mathbb{R}^{N}} u(x)$. We want to show that $u \equiv a$. If $u\left(x_{0}\right)=a$ for some $x_{0} \in \mathbb{R}^{N}$, then by condition $\left(F_{4}\right)$, a simple application of the strong maximum principle (see [Va] or [PS, Theorem 1]) shows that $u \equiv a$. Suppose now $u<a$ on $\mathbb{R}^{N}$. We are going to deduce a contradiction.

Let $x_{n} \in \mathbb{R}^{N}$ be such that $u\left(x_{n}\right) \rightarrow a$ as $n \rightarrow \infty$. Define $u_{n}(x)=u\left(x+x_{n}\right)$ and $c_{n}(x)=c\left(x+x_{n}\right)$ for $x \in \mathbb{R}^{N}$. Then

$$
-\Delta_{p} u_{n}=c_{n} f\left(u_{n}\right), x \in \mathbb{R}^{N} .
$$

Since $0 \leq c_{n} f\left(u_{n}\right) \leq c_{2} \max _{s \in[0, a]} f(s)$ and $0 \leq u_{n} \leq a$, by standard regularity theory (see [To]) we find that $\left\{\left.u_{n}\right|_{\Omega}\right\}$ is bounded in $C^{1, \alpha}(\Omega)$ for some $\alpha$ and any bounded smooth domain $\Omega$ of $\mathbb{R}^{N}$. Now $C^{1, \alpha}(\Omega)$ imbeds compactly into $C^{1}(\bar{\Omega})$. Thus $\left\{\left.u_{n}\right|_{\Omega}\right\}$ has a convergent subsequence in $C^{1}(\bar{\Omega})$. From this fact and a standard diagonal process, we can find a subsequence of $\left\{u_{n}\right\}$, say $\left\{u_{n_{k}}\right\}$, that converges to some $u^{*} \in C^{1}\left(\mathbb{R}^{N}\right)$ in the $C^{1}$ norm on any compact subsets of $\mathbb{R}^{N}$. Clearly we must have $0 \leq u^{*} \leq a$ and $u^{*}(0)=a$. 
Since $c_{1} \leq c_{n}(x) \leq c_{2}$, we have $-\Delta_{p} u_{n} \leq c_{2} f\left(u_{n}\right)$ and hence, by passing to the limit,

$$
-\Delta_{p} u^{*} \leq c_{2} f\left(u^{*}\right)
$$

Since $u^{*} \leq a$ and $u^{*}(0)=a$, we can now apply the strong maximum principle to conclude that $u^{*} \equiv a$. It follows in particular that $u_{n_{k}} \rightarrow a$ uniformly on any compact subsets of $\mathbb{R}^{N}$ as $k \rightarrow \infty$.

Since $\xi \in\left(p-1, \frac{N p}{N-p}-1\right)$, it is well known (see, for example, $[\mathrm{Dr}]$ ) that the problem

$$
-\Delta_{p} u=u^{\xi}, x \in B_{1}(0),\left.u\right|_{\partial B_{1}(0)}=0
$$

has a radially symmetric positive solution $u=u_{1}$ with $u_{1}(0)=\max _{x \in B_{1}(0)} u_{1}(x)$. Here, and in what follows, we use the notation $B_{r}\left(x_{0}\right)=\left\{x \in \mathbb{R}^{N}:\left|x-x_{0}\right|<r\right\}$.

Denote $\alpha=-p /(\xi-p+1)$ and $c=\left(c_{1} \sigma / 2\right)^{\alpha / p}$. Then for any $R>0$, it is easily checked that $v_{R}(x):=c R^{\alpha} u_{1}(x / R)$ satisfies

$$
-\Delta_{p} v_{R}=\left(c_{1} \sigma / 2\right) v_{R}^{\xi}, x \in B_{R}(0),\left.v_{R}\right|_{\partial B_{R}(0)}=0 .
$$

Clearly $v_{R}(x) \leq v_{R}(0)=c R^{\alpha} u_{1}(0)$. We now fix $R$ large enough such that $c R^{\alpha} u_{1}(0)$ $=\delta / 2$, where $\delta$ is given in $\left(F_{2}^{\prime}\right)$. Then $v_{R} \leq \delta / 2$ on $B_{R}(0)$.

We next show that $u(x) \geq \delta / 2$ over $\mathbb{R}^{N}$. To this end, we fix an arbitrary $x_{0} \in \mathbb{R}^{N}$. Since $u_{n_{k}}(x) \rightarrow a$ as $k \rightarrow \infty$ uniformly for $x \in B_{R}(0)$, we can find $m=n_{k}$ with large enough $k$ so that $u_{m}(x)>\delta$ on $B_{R}(0)$. We now make use of the weak sweeping principle. For $t \in[0,1]$ we define $\zeta_{t}(x)=x+t\left(x_{m}-x_{0}\right), \mathcal{D}_{t}=\zeta_{t}(\mathcal{D})$ with $\mathcal{D}=B_{R}\left(x_{0}\right)$. Clearly $\mathcal{D}_{0}=\mathcal{D}$ and $\mathcal{D}_{1}=B_{R}\left(x_{m}\right)$.

Since $-\Delta_{p} u \geq 0$ on $\mathbb{R}^{N}$, by the strong maximum principle we know that $u(x)>0$ for all $x$. By our previous assumption, $u(x)<a$ for all $x$. Hence there exists $\delta_{1} \in(0, \delta / 2)$ such that $a-\delta_{1} \geq u(x) \geq \delta_{1}$ for all $x$ in the compact set $\bigcup_{t \in[0,1]} \overline{\mathcal{D}}_{t}$. Define

Then clearly

$$
v_{t}(x)=v_{R}\left[x-x_{0}-t\left(x_{m}-x_{0}\right)\right]
$$

$$
-\Delta_{p} v_{t}(x)=\left(c_{1} \sigma / 2\right) v_{t}^{\xi}(x) \leq(1 / 2) c(x) f\left(v_{t}(x)\right), \forall x \in \mathcal{D}_{t}, \forall t \in[0,1] .
$$

Define $u_{t}=u$ for $t \in[0,1]$. Since $a-\delta_{1} \geq u(x) \geq \delta_{1}$ for $x \in \bigcup_{t \in[0,1]} \overline{\mathcal{D}}_{t}$, we can find $\epsilon \in\left(0, \delta_{1}\right)$ such that $\left(c_{1} / 2\right) f(u(x)) \geq \epsilon$ for all such $x$. Hence,

$$
\begin{gathered}
-\Delta_{p} u_{t}=c(x) f\left(u_{t}\right) \geq(1 / 2) c(x) f\left(u_{t}\right)+\epsilon, \forall x \in \mathcal{D}_{t}, \forall t \in[0,1], \\
u_{t}(x) \geq \delta_{1} \geq v_{t}(x)+\epsilon, \forall x \in \partial \mathcal{D}_{t}, \forall t \in[0,1] .
\end{gathered}
$$

Note also that on $\mathcal{D}_{1}=B_{R}\left(x_{m}\right), u(x) \geq \delta$ and $v_{1}(x) \leq \delta / 2$. Therefore, we can use Lemma 2.7 to conclude that

$$
u(x) \geq v_{t}(x), \forall x \in \mathcal{D}_{t}, \forall t \in[0,1] .
$$

In particular, $u(x) \geq v_{0}(x)$ on $\mathcal{D}_{0}=B_{R}\left(x_{0}\right)$ and $u\left(x_{0}\right) \geq v_{0}\left(x_{0}\right)=v_{R}(0)=\delta / 2$. As $x_{0} \in \mathbb{R}^{N}$ is arbitrary, this implies that $u(x) \geq \delta / 2$ on $\mathbb{R}^{N}$. It now follows from Lemma 2.3 of $\left[\mathrm{DG}\right.$ ] that $u(x) \geq a$ on $\mathbb{R}^{N}$. Hence $u \equiv a$. But this contradicts our previous assumption that $u<a$ on $\mathbb{R}^{N}$.

Proof of Theorem 2.4. Suppose that $u$ is a solution to (2.2) satisfying $0 \leq u \leq a$. We may assume that $\|u\|_{\infty}<a$ for otherwise $u \equiv a$ by Proposition 2.6. By our assumption we can write $f(u)=u^{\xi} g(u)$ with $g(u)$ positive, $C^{1}$ and nonincreasing in $(0, a)$. Now we define a $C^{1}$ function $\tilde{g}(u)$ for $u>0$ such that

$\tilde{g}(u)=g(u)$ for $u \in\left(0,\|u\|_{\infty}\right]$, 
$\tilde{g}(u)=$ positive constant for $u>(1 / 2)\left(\|u\|_{\infty}+a\right)$,

$\tilde{g}^{\prime}(u) \leq 0$ for $u \in\left(\|u\|_{\infty},(1 / 2)\left(\|u\|_{\infty}+a\right)\right)$.

Then clearly $\tilde{f}(u):=u^{\xi} \tilde{g}(u)$ satisfies the conditions (i)-(iii) of Proposition 2.5, and $u$ solves $-\Delta_{p} u=\tilde{f}(u)$. It follows that $u \equiv 0$.

Remark 2.9. By using a more involved proof for Proposition 2.6, condition $\left(F_{2}^{\prime}\right)$ can be removed. As this point does not improve our main result, Theorem 2.4, we only give a brief sketch of its proof here. The idea is to construct the lower solutions $v_{t}$ in a different way. Indeed, let $u>0$ be a solution of (1.2) as given in Proposition 2.6. By minimizing the corresponding functional of the problem

$$
-\Delta_{p} v=\lambda f(v),\left.v\right|_{\partial B_{1}(0)}=0
$$

over the order interval $\left[0,\left.u\right|_{B_{1}(0)}\right]$ one finds that it has a radial solution $v$ satisfying $0<v \leq u$ provided that $\lambda$ is large enough. Then $v_{t}$ can be obtained by a rescaling and shifting of $v$ much as before.

Remark 2.10. The conclusion of Proposition 2.6 can be strengthened. We can show that there is a positive $\delta$ such that any nonconstant solution in Proposition 2.6 satisfies $u(x) \leq a-\delta$ on $R^{N}$. This is useful for studying solutions on bounded domains when the diffusion rate is small. Note also that the results here could be used to simplify the proof of Theorem 4 in [Da].

Let us end this note with some discussions of a simple yet illustrative example. From Theorem 2.4 and Remark 2.2 (i), we immediately see that when $1<p \leq 2$, if $\xi \in\left(p-1, \frac{N p}{N-p}-1\right)$ and $q>\xi$, then the only nonnegative entire solutions of

$$
-\Delta_{p} u=u^{\xi}-u^{q}
$$

are $u \equiv 0$ and $u \equiv 1$. The restriction that $p \leq 2$ is due to condition $\left(F_{4}\right)$; we believe that this restriction can be removed. Note that if $\xi>\frac{N p}{N-p}-1$ and $q>\xi$, then it is known that (2.5) has radial ground state solutions (see [KMPT] for the case $p=2$ and [T] for the general case $p>1$ ). When $\xi=\frac{N p}{N-p}-1$ and $q>\xi$, it follows from [T Theorem (i)] that (2.5) has no radial ground states. It would be interesting to know whether there can be other types of nonconstant positive solutions in this latter case.

In the case $p=2$, this question has a negative answer. Indeed, by Theorem 3 of Bianchi [B] , if for $s \in(0, \infty)$, the function $g(s)$ is locally Lipschitz, is nonnegative and $g(s) / s^{(N+2) /(N-2)}$ is nonincreasing, then any positive $C^{2}$ solution of $-\Delta u=$ $g(u)$ in $\mathbb{R}^{N}(N>2)$ is either a constant and $g$ vanishes on that constant, or

$$
u(x)=\frac{k}{\left(\left|x-x_{0}\right|^{2}+h^{2}\right)^{(N-2) / 2}}
$$

for some $x_{0} \in \mathbb{R}^{N}$ and positive constants $k$ and $h$, and $g(s)$ is a suitable scalar multiple of $s^{(N+2) /(N-2)}$ for $s \in\left(0, \max _{\mathbb{R}^{N}} u\right]$.

If we take $g(s)=s^{(N+2) /(N-2)}-s^{q}$ with $q>(N+2) /(N-2)$ for $s \in[0,1]$ and $g(s)=0$ for $s>1$, then Bianchi's result implies that

$$
-\Delta u=u^{(N+2) /(N-2)}-u^{q}
$$

has no solution in $\mathbb{R}^{N}$ satisfying $0<u \leq 1$ except $u \equiv 1$. On the other hand, by Remark 2.2 (i), we know that any positive solution of (2.6) satisfies $0<u \leq 1$. Thus $u \equiv 1$ is the only positive solution of (2.6). 


\section{REFERENCES}

[AW] D.G. Aronson and H.F. Weinberger, 'Multidimensional nonlinear diffusion arising in population genetics', Advances in Math., 30(1978), 33-76. MR 80a:35013

[B] G. Bianchi, 'Non-existence of positive solutions to semilinear elliptic equations on $R^{n}$ or $R_{+}^{n}$ through the method of moving planes', Comm. Partial Diff. Eqns., 22(1997), 1671-1690. MR 98g:35063

[BP] M. Bidaut-Veron and S. Pohozaev, 'Non-existence results and estimates for some nonlinear elliptic equations', J. Anal. Math., 84(2001), 1-49. MR 2002f:35085

[Da] E.N. Dancer, 'On the number of positive solutions of weakly nonlinear elliptic equations when a parameter is large', Proc. London math. Soc., 53(1986), 429-452. MR 88c:35061

[D] J. Diaz, Nonlinear Partial Differential Equations and Free Boundary Problems, Vol. 1, Elliptic Equations, Pitman research notes in math., Vol. 106, Boston, 1985. MR 88d:35058

[Dr] P. Drabek, 'Nonlinear eigenvalue problems and Fredholm alternative', Nonlinear Differential Equations, Ed. P. Drabek et al., Chapman \& Hall/CRC, London, 1999. MR 2000e:35057

[DG] Y. Du and Z. Guo, 'Liouville type results and eventual flatness of positive solutions for p-Laplacian equations', Advances Diff. Eqns., 7(2002), 1479-1512.

[DM] Y. Du and L. Ma, 'Logistic type equations on $\mathbb{R}^{N}$ by a squeezing method involving boundary blow-up solutions', J. London Math. Soc., 64(2001), 107-124. MR 2002d:35089

[KMPT] M.K. Kwong, J.B. McLeod, L.A. Peletier and W.C. Troy, 'On ground state solutions of $-\Delta u=u^{p}-u^{q}$, J. Diff. Eqns., 95(1992), 218-239. MR 93d:35045

[PS] P. Pucci and J. Serrin, 'A note on the strong maximum principle for elliptic differential inequalities', J. Math. Pures Appl., 79(2000), 57-71. MR 2001g:35277

[S] J. Serrin, 'Nonlinear elliptic equations of second order', Lectures at AMS Symposium on Partial Differential Equations, Berkeley, 1971.

[SZ] J. Serrin and H. Zou, 'Cauchy-Liouville and universal boundedness theorems for quasilinear elliptic equations and inequalities', Institut Mittag-Leffler report No. 34, 1999/2000.

[T] M. Tang, 'Uniqueness and global structure of positive radial solutions for quasilinear elliptic equations', Comm. Partial Diff. Eqns., 26(2001), 909-938. MR 2002f:35093

[To] P. Tolksdorf, 'Regularity for more general class of quasilinear elliptic equations', J. Diff. Eqns., 51(1984), 126-150. MR 85g:35047

[Va] J.L. Vazquez, 'A strong maximum principle for some quasilinear elliptic equations', Appl. Math. Optim., 12(1984), 191-202. MR 86m:35018

School of Mathematics and Statistics, University of Sydney, New South Wales 2006, Australia

E-mail address: normd@maths.usyd.edu.au

School of Mathematics, Statistics and Computer Science, University of New England, Armidale, New South Wales 2351, Australia

E-mail address: ydu@turing.une.edu.au 